\title{
Generating a Coherent Text Describing a Traffic Scene
}

\author{
Hans-Joachim Novals ${ }^{1}$ \\ Fachbereich Informatik, Universität Hamburg \\ D-2000 Hamburg 13, West-Germany
}

\begin{abstract}
If a system that embodies a reference semantic for molion verbs and prepositions is to generate a coherent text describing the recognized motions it needs a decision procedure to select the events. In NAOS event selection is done by use of a specialization hierarcley of motion verbs. The strategy of axticipated visualization is used for the selection of optional deep cases. The system exhilhits low-level strategies which are based on verbinherent properties that allow the generation of a coherent descriptive text.
\end{abstract}

\section{1 lntroduction}

This contribution focuses on the verbalization component of the NAOS system (the acronym stands for NAtural language description of Object movements in a traffic Scene). NAOS is designed to explore the border area between computer vision and natural language processing, especially the realm of recognizing and verbalizing motion concepts in image sequences.

NAOS goes all the way from a representation of a real-world trafic scene to a natural language text describing the scene.

The representation of the scene basically consists of its geometry (therefore called geometric seene description (GSD)). To give an impression of the representation a GSD contains for each frame of the image sequence:

o instance of time
visible objects
o viewpoint
- illumination
- 3D shape
- surface characteristics (color)
- class
- identity
- 3D position and orientation in each frame

(for a detailed description of the GSD see $\mid 16]$ )

For everit recognition we use cvent models ([18], [19]) which define a reference semantic for motion verds. In the current implementation of the NAOS system about 35 motion verbs and the prepositions beside, by, in-front-of, near, and on may be recognized by matching the event models against the representation of the scene.

In this paper we are neither concerned with the representation of the underlying scene data nor with the question of cvent recognition as these issues have been published elsewhere (see [16] [17] [20]). Instead, we focus on the generation of a coherent text describing the image sequence.

In the next section we briefly describe the representation of the recognized events which form the initial datia for the verbalization component. Then the overall strategy for composing a cohberent, description is discussed. The following section introduces at partial solution to the selection problem which is based on the stratogy of anticipated visualization. Fourth, we show how some linguistic choices like passive, restrictive relative clanses, and negation

IT thank B. Neumann who contributed several ideas to this article. are natural consequences of the task of generating unambiguous referring expressions. In the last section we relate our research with current work on language generation.

\section{Initial Data}

Verbalization starts when event recognition has been achieved. Besides complex events like ovortake and tum off, other predi. cates like in-fronti-of, besides, move, ete are also instantiated. Below is a section of the database after event recognition has taken place (the original entries are in German).

1: (MOVE PERSON 1040 )

2: (WALK PERSON1 0 40)

3: (RLCEDL PLRSON1 FBI 2040 )

4: (OVERTAKE BMW1 VW1 (10 12) (15 32))

5: (MOVH BMW1 1040 )

6: (IN-JRON'T-OF VW1 TRAFIIC-LGGF'I 27 32)

The above entries are instantiations of event models containing symbolic identifiers for scene objects (e.g. BMWl). The last iwo elements of an instantiation denote the start and end time of the event.

We use the following notations to denote the event lime:

1. (...Tb Te)

2. $\left(\ldots\left(T b_{\min } T b_{\max }\right)\left(T e_{\min } T \varepsilon_{\max }\right)\right)$

3. $\left(\ldots\left(T b_{\min } T b_{\max }\right) T e\right)$

4. $\left(\ldots T b\left(T e_{\min } T e_{\text {max } x}\right)\right)$

Tb, Te denote start and end time of an event. The first. notation is used for durative events (o.g. novo). A durative event is also valid for each subinterval of (T' T Te)

The second notation is used for non-durative eventis (o.g. overtake). Start and end time of such an event are both restricted by lower and upper bounds. Note, that ron durative events are not valid for each subinterval of the event boundaries.

The third notation is used for remaltativas events (e.g. siop) The start time of a resuldative event lies within an interval whereat the end time is a time-point.

Finally, the last notation is used for inchoative events (e.g. start moving, corresponding to the German verb losha h. aren). Inchostive events have a well defined start time whereas the end time lies within an interval.

For the task of generating a coherent description of a brafic scene NAOS first instantiates all event models and predicates which may be instantiated using the scene data. This leads to the well known selection problerin of natural language gencration. For one object there may be many instantiations with different time intervals, hence the task of the verbalization component to choose what to say. In the next section we discuss the theoretical background on which our verbalization component is based.

\section{\& Theoretical Background}

In general, language is not generated per se but is always in." tended for a hearer. Furthermore, language is used to fulfil certain 
goals of the speaker which may sometimes simply be to inform the hearer about certain facts.

In NAOS the generation of a description of the underlying inage sequence aims at diminishing the discrepancy between the system's knowledge of the scene and the hearer's knowledge (the sane motivation is used in Davey's program [6]). Concernig the hearer we make the following assumptions:

1. S/he knows the static background of the scene, i.e. the streets, houses, traffic lights, etc.

2. $S /$ he did not utter specific interests except: Describe the scene?

$A$ description may be the result of such diverse speech acts as TNFORM, DROMISE, PERSUADR, or CONVINCW. NAOS only generates the spech act INHORM

To inform a hearer about something moans to tell her/him something $\mathrm{s} /$ he has not known before, something that is true and new. In NAOS the definition of true utterances builds on the situational semantics of Barwise and Perry [3|. They understiand the meaning of an utterance as a relation between the utterance and the described situation. The interpretation of an utterance by a hearer usually consists of a set of possible situations with a meaning relation to the utterance. We now define an utterance to be true if the set of possible situations contains the actually occurred situation.

The requirement to generate true utterances has two consequences for our verbalization component. First, the verbalization process must take the hearer's meaning relations into account. This co. incides with the communication rule to tune one's utterances to the hearer's comprehension ability. Second, assuming that the speaker has the sane meanng relations as the hearer, the speaker can anticipate the hearer's interpretation of an 1 itterance, i.e. the possible situations implied solely by the utterance can be generated without knowledge of the actual situation. In the case of scene descriptions these situations are equivalent to the hearer's visualization of an unknown scene.

An utterance must be new to the hearer in order to inform hirm. In the context of situational semantics we define an utterance to be new if its interpretation restricts the sot of possible situations implicd by previous utterances. Thus new information additionally specifies described situations.

The task of a verbalization component is to choose utterances such that they inform in the above sense. Therefore it is necessary to anticipate the hearer's understanding for judging whether a planned utterance carries now information.

T.he general principle for hearer simulation is depicted in figure 1 .

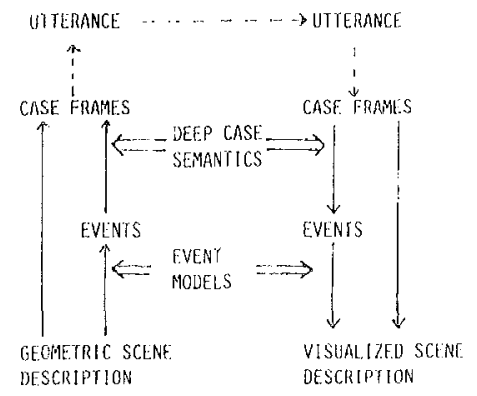

SPEAKER MFARER

I'igure 1: Mearersimulation
On the side of the speaker the event recognition process leads by use of event models to instantiated event morlels (called events in the figure). $\Lambda$ first selection process chooses arnong the instantiations those which are to be verbalized. As event models are associated with verbs the appropriate case frame of the verb is available. A second selection process now chooses anong the optional deep cases of the verb. This is where the deep case semantics comes into play. If, for instance, it is decided that a locative expression should be generated it is necessary to know how the location of an object may be expressed in natural language as in the geometric: scene description the location of an olject is given by its $x, y$, and z coordinates. The deep case semantics also contcins information about the prepessitions which may be used for cxpressing a specific deep case

Assuming that the hearer has the same meaning relations as the speaker he basically can use the speaker's processes in reversic order and reconstruct the underlying case frame from the utterance and thus build a visualized scene description.

Note, however, that we agree with Olson [21] that the verbali. zation of a visual event always leads to a loss of information. In our case, for instance, we cannot assume that the hearer knows the $x$, $y$, and \% coordinates of an object when he hears the phase in front of the department of computer science. Such a phrase generates is set of coordinates defining the region which corresponds to the preposition in-front-of. The actual foceation of the object which give rise to the generation of the phrise lies somewhere within that region. Presently, hearer modeling stops at the level of case frames and the visualized scene is anticipated (see section 4.2).

As shown in figure 1 the case frame of a verb plays a central role in our verbalization component. We adopt the view of tillmore expressed in his scenes-and frames semantic (7) that case frames relate scenes to natural language expressions.

\section{The Selection Troblern}

Usually this problem is divided into the subtasks of deciding what to say and how tos say it. Asi mentioned above NAOs uses two selection processes. First, it selects among the instantiated events and second, it selects arnong the optional decp cases of the verb associated with the chosen cvent. The first selection process corresponds to deciding what to say and the second one determines largely how to say it as will be shown later.

The selection processes are based on the representation of the case semantics of an event, model and on a specialization hierarchy of the verbs. Below is the representation of the case semantics for the event model ïberholen (overtake).

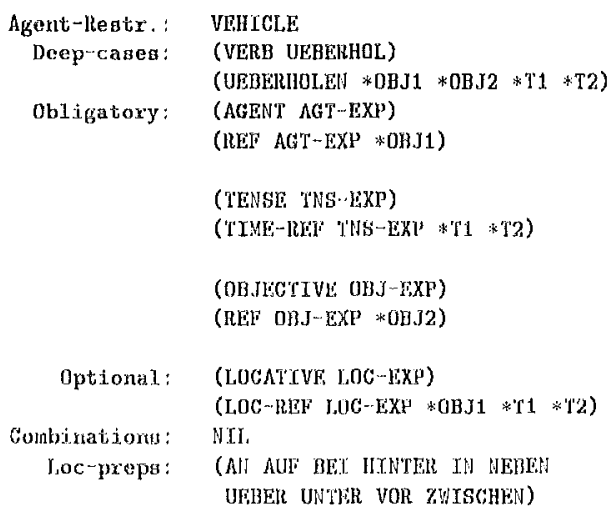

The first slot specifies the agent restriction. The deepecases slot 
contains first the verb stem of überholen as needed by the generation component and second the formal notation for an instantiation. The obligatory cases must be generated but may be omitted in the surface string in case of elliptic utterances whereas optional deep cases need not be generated at all. In the combinations slot it is represented which deep cases may be generated together (e.g. for the verb fahren (drive) it is not allowed to generate a single SOURCE but instead SOURCE and GOAL must be generated). The Loc-preps slot specifies the prepositions which may be used with the verb iiberholen to generate locative expressions.

The case descriptions in the obligatory and optional slots consist of two parts: a declaration of an identifier for the case expression on the language side, and a predicate (in general a list of predicates) relating the case expression to the scene data. The most important predicates are REF, TIME-REF, and LOC-REF.

REF generates referring phrases for internal object descriptors like BMW1. TIME-REF generates the tense of the verb. As descriptions are usually given in present tense, presently TIME-REF only generates this tense. LOC-REF relates the abstract location of the object as given by its coordinates to a natural language expression for a reference object. Note, that REF has to be used to generate a referring phrase for the reference object. Consider the sixth entry of the database in section 2. The instantiation only contains internal identifiers for objects, like traffic-light1, for which referring phrases have to be generated (see section 4 for further details on REF).

In NAOS we use a specialization hierarchy for motion verbs. This hierarchy is pragmatically motivated and is rooted in situational semantics. It is no hierarchy of motion concepts as the one proposed in [23]. It connects general verbs with more special ones. A situation which may be described using a special verb implies the application of all more general verbs. Take for instance the verb iiberholen (overtake). It implies the use of the more general verbs vorueberfahren, vorbeifahren (drive past), passieren (pass), naehern-r (approach), entfernen-r (recede), fahren (drive, move), and bewegen-r (move)

It should be intuitively plausible that such a hierarchy is also used for event recognition. If, for instance, no naehern-r (approach) can be instantiated the more special events need not be tested.

\subsection{Event Selection}

In NAOS the overall strategy for generating a descriptive text is as follows:

- Group all moving objects according to their classmembership;

- For each object in each group describe the motions of the object for the time interval during which it was visible in the scene.

Event selection for an object is done according to the following algorithm:

1. Collect all events in the interval where the object was visible and where the object was the agent;

2. determine for each timepoint during the object's visibility the most special event of the above collected ones;

3. if two events have the same specificity then either take the one which started earlier and has the same or longer duration as the other one or take the one with longer duration;

4. put the selected events on the verbalization list of the object in temporally consecutive order.

Consider the following example. All events which were found for PERSON1 are:

\section{(RECEDE PERSOH1 PBI 20 40) (ENTFERNEN-R PERSOH1 FBI 20 40) \\ (WALK PERSON1 0 40) \\ (GEHEN PERSONI 0 40) \\ (MOVE PERSON1 0 40) \\ (BEWEGEN-R PERSOH1 0 40)}

The above algorithm leads by use of the specialization hierarchy to the following verbalization list for PERSON1:

( ( (WALK PERSON1 O 40) (0 20))

((RECEDE PERSON1 FBI 20 40) (20 40))

(The last entry in parenthesis of each selected event denotes the interval in which the event was the most special one.)

\subsection{Selection of Optional Deep Cases}

This selection process is our first implementation of the strategy of anticipated visualization. The underlying question is: Which optional deep cases should be selected to restrict the hearer's possibilities of placing the trajectory of an object in his internal model of the static background of the scone?

In NAOS the selection algorithm answering the above question is rather straightforward. It is based on the manner of action of the verb, the verbtype, and the hearer's knowledge. The algorithm is graphically represented in figure 2 .

\begin{tabular}{|c|c|c|}
\hline EVENTTYPE & VERBTYPE & DEEP CASES \\
\hline \multirow{3}{*}{ NON-DURATIVE, INCHOATIVE, } & DIR & LOCATIVE? \\
\hline & LOC & DIRECTION?, LOCATIVE? \\
\hline & REO & DIRECTION? \\
\hline \multirow[t]{2}{*}{ RESULTATIVE } & $\overline{\mathrm{DIR}, \mathrm{LOC}}$ & LOCATIVE? \\
\hline & REO & NIL \\
\hline \multirow{3}{*}{$\begin{array}{c}\text { DURATIVE } \\
T_{t, 0}=S B \wedge T_{\text {end }}=S E\end{array}$} & REO & NIL \\
\hline & DIR, STAT & LOCATIVE? \\
\hline & LOC & DIRECTION?, LOCATIVE? \\
\hline \multirow{3}{*}{$T_{b e v}=S B \wedge T_{c n d} \neq S E$} & STAT & LOCATIVE? \\
\hline & DIR, REO & NIL \\
\hline & LOC & DIRECTION? \\
\hline \multirow{4}{*}{$T_{\text {ted }} \neq S B \wedge T_{\text {end }}=S E$} & S'TA'T & LOCATIVE? \\
\hline & DIR & LOCATIVE? \\
\hline & LOC & SOURCE?, DIRECTION? \\
\hline & REO & NIL \\
\hline \multirow{3}{*}{$T_{\log g} \neq S B \wedge T_{e n d} \not S E$} & $\mathrm{REO}$ & NIL \\
\hline & DIR, STAT & LOCATIVE? \\
\hline & LOC & SOURCE?, GOAL? \\
\hline
\end{tabular}

Figure 2: Selection of Deep Cases

The abbreviations denote: $T_{b e j}, T_{e n d}$ start, end time of the event; SB, SE: scene begin and scene end; IIR, LOC, STAT, REO: directional (turn off, retırn), locomotion (walk, overtake), and static (stand, wait) verbs, finally verbs whose recognition implies reference objects (reach s. th., arrive at).

The figure has to be read as follows. If an inchoative event like losfahren (start moving) has to be verbalized which has the verbtype locomotion, then choose direction? and locative? as deep cases. The question mark generally means, look into the partnermodel to see whether this deep case has already been generated for another event. If so, determine by use of the object's actual location (represented in the scene representation) whether it is still valid. If this is the case don't generate a natural language expression for this deep case, otherwise do.

Presently the partnermodel contains information about the static background of the scene and about what has been said so far in the same relational notation as was shown for instantiations in section 2. It is being updated when an event is verbalized.

Note, that for durative events the decision is based on whether the start and end time of the event coincide with the beginning or ending of the image sequence. Consider the first case for durative events as given in figure 2. Right from the beginning of the sequence there is a car moving along a street until the sequence ends. In such a case it is not possible to verbalize a source as the object may have started its motion anywhere. To restrict the hearer's visualization, 
direction and locative cases are verbalized, leading to a sentence like: The car moves on Schlüterstrent in direction of Hallerplace. Verbalizing a direction when the static background is known restricts the trajectory to being on one side of the road. Basically, our direction case is a goal or source case where only two propositional phrases are allowed, the German phrases in Richtung and aus Richtung (in direction, from direction). These phrases do not imply that the motion ends at the goal location as do most prepositional phrases in German which have to be in accusative surface case to denote a goal. The English language is in this respect inherently ambiguous. In the sentence The car moves behind the truck, the phrase behind the truck may denote a locative or goal deep case. In German these cases are distinguished at the surface. For locative the above sentence translales to Das Auto fährt hinter dem $L K W$, for the goal case, it translates to Das Auto fährt hinter den $L K W$.

We have to distinguish different verbtypes as e.g. the meaning of a directional plirase changes with the verbtype. Consider the sentences The car moves in direction of Hallorplace versus The car stands in direction of Nallerplace (in German both sentences are well formed). The first sentence denotes the direction of the motion whereas the second one denotes the orientation of the car. We thus distinguish between static (STA'V) and locomotion (LOC) verbs. The third verbtype, directional (DIR), is used for verbs with a strong directional component like umkehren (return), abbiegen (turn off), etc. $\Lambda$ s they already imply a certain direction the additional verbalization of a direction using a prepositional phrase does usually not lead to acceptable sentences. The fourth type (REO) is used for verbs like erreichen (reach s. th.) having an obligatory locative case.

The main result to note here is that the selection processes are low-level and verboriented. The only higher level goal is to inform the hearer and to convey as much information about an event as possible. In the next section we show by different verbalizations of the same scene how rather complex syntactic structures arise.

\section{Generation}

The general scheme for the generation process is as follows:

1. Sort the objects according to their classmembership, vehicles first, then persons;

2. in the above partial order sort the objects according to their time of occurrence in the scene, earliest first;

3. do for all clements in each verbalization list of each object

(a) if the current event has a precedent and its event time is included in the precedent's, begin the sentence with dabei (in the meantime); go to (c);

(b) if the current event has a precedent and its event time overlaps the precedent's, begin the sentence with unterdessen (approx. in the meantime); go to (c);

(c) determine the optional deep cases and build a simple declarative sentence by using all chosen deep cases and applying the deep case semantics.

Two temporally consecutive events are not verbalized using a temporal adverb as in the cases of inclusion and overlapping. This is due to the fact that from the linear order of the sentences the hearer usually infers consecutivity.

The result of the above algorithm is a formal representation of the surface sentence which, roughly, contains the verb's stem, genus verbi, modality, and person, all deep cases in landorn order, and all stems of the lexical entries which appear in the surface sentence. 'This representation is taken as input by the system SUTRA (for further details on the formal representation and the SUTRA system see [4|) which then generates a correctly inflected German sentence. Below is an example of the output of NAOS.

18. ,ausgabe text

DIE SZENE ENTHAELT VIER BEWEGTP OBJEK'TE: DREI PKWS UND EINEN FUSSGAENGER.

The scene consists of four moving objects: three vehicles and a pedestrian.

EIN GRUENTR VW NAEHERT SICH DEM GROSSEN FUSSGAENGER AUS RICHTUNG HALLERPLATZ. ER FAEHR'T AUF DER SCHLUETERSTRASSF.

A green $V W$ approaches the tall pedestrian from the direction of $\mathrm{Hal}$. lerplace. It drives on Schlueterstreet.

EIN GELBER VW FAEHRT VON DER ALTEN POST VOR DIE AMPEL. WALHRFNDDESSEN ENTFERN'T ER SICH VON DEM GRUENEN VW.

$A$ yellow $V W$ drives from the old postoffice to the traffic light. In the mentime it recedes from the green $V W$.

EIN SCHWARZER BMW FAEHRT IN RICHTUNG HALLERPLAT\%. DABEI ULBERHOLT LR DIN GELBEN VW VOR DEM FACIIBJREICH INFORMATIK. DER SCHWARZE BMW LNTIERNT SICII VON DEM GRUENEN VW

A black $B M W$ drives in the direction of Hallerplace. During this time it overtakes the yellow $V W$ in front of the department of computer science. The black $B M W$ recedes from the green $V W$.

DER GROSSE FUSSGAENGER GEHT IN RICHTUNG DAMMTOR AUF DEM SUEDLICHEN FUSSWEG WESTLICH DER SCHLUETEISTRASSF. WALHRPNDDESSEN ENTFERNT ER SICH VON DEM FACHBEREICH INFORMATIK.

The tall pedestrian walks in the direction of Danmer on the southern sidewalk west of Schlueterstrect. In the meantime he recedes from the department of computer science.

19. ,logout

The first sentence above is a standard one having the same structure for all different scenes. The remaining four paragraphs are motion descriptions for the four moving objects.

We now discuss step (c) of the above algorithm in more detail as it covers some interesting phenomena.

Consider the third paragraph describing the motions of the yellow VW. The verbalization list for this object is:

(( (DRIVE VW1 $10 \quad 20) \quad\left(\begin{array}{lll}10 & 25))\end{array}\right)$

((RECEDE VW1 VW2 25 32) $(25$ 32) ))

The beginning (SB) and ending of the sequence (SE) lie at points 0 and 40, respectively. According to the selection algorithm (figure 3) a SOURCE should be verbalized for a durative event with the above event time if the verbtype is LOC. The generation algoritlm checks whether the chosen optional cases are allowed for the verb, if so, it is further checked whether the combinations are allowed. As a SOURCE may not be generated alone for a fahren (drive, move) event, SOURCE and GOAL are generated.

The fourth paragraph shows the outcome of a deep case selection in which the chosen case is not allowed for the verb. The verbalization list for the black BMW contains only überbolen (overtake) and entfernen-r (recede).

(( (OVERTAKE BMW1 VW1 (10 12)(12 32) (10 32)) ((RECEDE BMW1 VW2 20 40) (32 40)))

According to event- and verbtype DIRECTION is chosen as the appropriate deep case. As this case may not be used with the verb overtake two sentences are generated, one describing the direction 
of the motion and the other one describing the specific event. The second sentence begins with a temporal adverb specifying that both motions occur at the same time. In order to generate the two sentences first the classmembership of the agent of the verb which may not take the chosen deep case is determined. Then the specializationhierarchy is used to go up to either fahren (drive, move) or gehen (walk) as those verbs may take any deep case. Then the sentences are generated.

Consider the following verbalization list:

(((OVERTAKE BMW1 VW1 (0 8) (12 18) ( 018$))$

((DRIVE BMW1 O 40) (18 40)))

Assuming the direction and location of the motion to be the same as before the algorithm presented so far would generate $A$ black $B M W$ drives in the direction of Hallerplace. During this time it overtakes the yellow $V W$ in front of the department of computer science. The black $B M W$ drives.

According to the deep case selection algorithm a DIRECTION and LOCATIVE should be generated for the second event above. $\Lambda \mathrm{s}$ both cases have already been generated with the first event and are still valid the sentence The black $B M W$ drives is not generated because before generating a sentence it is checked whether the information is already known to the partner.

\subsection{Referring Phrases}

In this section some aspects of the referring phrase generator are discussed. As can be seen from the example text objects are characterized by their properties, introduced with indefinite noun phrases when they are not single representatives of a class and they may also be pronominalized to add to the coherence of the text. Therefore we use standard techniques as e.g. described in [8], [9].

We want to stress one aspect of our referring phrase generator, namely its capability to generate restrictive relative clauses with rnotion verbs. As it may be easily the case that a scene contains two objects with similar properties the task arises to distinguish them and generate unequivocal referring expressions.

It is an interesting fact, that we have several options to cope with this problem which each have their consequences.

One option is to adopt McDonald's scheme of generation without precisely knowing what to say next $[13]$. According to this scheme two similar objects are characterized in the following way in NAOS. When the first one is introduced it is characterized by it's properties e.g. a yellow $V W$. When the second one has to be introduced, REF notices that a yellow VW is already known to the partner and generates the phrase another yellow VW. It starts getting interesting in subsequent reference. The objects are then characterized by the events in which they were involved earlier whether as agent or in another role. This leads to referring phrases like the yellow $V W$, which receded from the pedestrian or the yellow $V W$, which has been overtaken. Note, how passive relative clauses arise naturally from the task of generating referring phrases in this paradigm. The same is also true for negation. Consider the case where the first yellow VW, say VW1, has passed an object and the second yellow VW, say VW2, has overtaken an object and both events are already known to the partner. If REF has to generate again a referring phrase for $V W 1$ it notices that pass is a more general verb than overtake and may thus also be applied for the overtake event. It therefore generates the phrase the yellow VW, which has not overtaken the other object to distinguish it unequivocally from VW2.

Below is an example of this strategy in a text for the same scene as above. The difference to the first scene is that we replaced the green $\mathrm{VW}$ by a yellow one.

10. , ausgabe text,
DIF SZENE LN'TH $A$ EL' VIER BEWEGTE OBJEKTE: DREI PKWS UND EINEN FUSSGAENGISR.

The scene consists of four moving objects: three vehicles and a pede. strian.

EIN GELBER VW NAEHERT SICH DEM GROSSEN FUSSGAENGER AUS RICITTUNG HALLERPLATZ. ER FAEHRT AUF DER SCHLUETERSTRASSE.

A yellow $V W$ approaches the tall pedestrian from the direction of Hallerplace. It drives on Schliteterstreet.

EIN ANDERER GELBER VW FAEIIT' VON DER ALTISN POST VOR DIE AMPEL. WAEHRENDDESSEN ENTTERNT ER SICH VON DEM GELBEN VW, DER SICH DEM GROSSEN FUSSGAENGIS GENAEHERT' HAT.

Another yellow VW drives from the old post oflice to the traffic light. In the meantime it recedes from the yellow $V W$ which approached the tall pedestrian.

EIN SCHWARZER BMW FAEHRT IN RICHTUNG HALLERPIA'TZ. DABEI UEBERHOLT ER DEN ANDEREN GELBEN VW, DER SICII VON DEM GELBEN VW ENTPERNT HAT, VOR DEM FACHBEREICH INPORMA'ITK. DER SCHWARZIE BMW ENTFERNT SICH VON DEM GLILBEN $V W$, DER NICHT UEBERHOLT WORDEN IST.

A black BMW drives in direction of Hallerplace. During this time it overtikes the other $V W$ which receded from the yollow $V W$ in thont of the department of computer science. The blick BMW recedes from the yellow $V W$ which was not overtaken.

DER CrROSSE FUSSGAENG ISR GHT IN RLCHTUNG DAMMTOR AUF DEM SUEIDHCHEN FUSSWEG WESTLYCH DHR SCIUUE'WRSTRASSE. WA HHRENDDESSEN EN'FERNT ER SICH VON DEM FACHBEREICH INFORMATIK.

The tall pedestrian walks in direction of Dammitor on the southern sidewalk west of Schlucterstreet. In the meantime he recedes from the department of computer science

11. ,logout

The consequences of this first option are rather complex syntactic structures which are not notivated by higher level stylistic choices.

let us now look at a second option which has also been implemented. Experience with the above algorithen for different scenes showed, that if more than two simblar objects are in a scene the restrictive relalive clauses becone hardly understandable. We thus determine how many similal objects there are in the scene belore we start the generation process. If there are more than two, REF generates names for them and introduces them as e.g. the first yellow $V W$, the second yellow $V W$ and so on and uses these phrases in subsequent references. An example of this strategy would look like the first example text where the different vehicles are named the first ..., the second .... The rest of the text would remain the same.

Taking this option implies leaving McDonald's scheme and approaching to a planning paradigm.

It should be noted here that there is a third option which has hardly been investigated, narnely to switch from contextual to cotextual reference as in phrases like the VWI mentioned last. We need further research before we can use such techniques effectively.

\section{Conclusion and Related Research}

We have proposed the scherne of anticipated visualization to generate coherent texts describing real-world events (visual data). The selection algorithrns are hased on low-level, verbinherent pro. perties, and on a pragmatically motivated verb hierarchy. 'Together with the verbalization component the NAOS system is now fully operational from event recognition to text genemation in the do- 
main of traffic scenes. As this domain is rich onough to still pose a lot of problerns this opens up the opportunily to integratio higher level strategies for e.f. combining sentences, selecting events, generating deictic expressions, etc.

The main difference between NAOS and other systerns for language generation is that we approach the verbalization problem from the visual side and thus are led to use basic selection algorithms. Other sysiems like 'TALESP'IN |15], KOS [12], 'TEXT |14, KAMP $[U]$, and HAM-ANS $\{10]$ start their processing with langunge whereas NAOS starts with images. II close connection to our rescarch is the work of $[2|| ,24|| 23],,[22 \mid$, and $|5|$. The first four authors deal with questions of motion recognition and with a re-. ference semantic: for motion verbs but are net concerned with text generation. 'They showed that case frames can be used to generate sinfle utierances. Conklin and Mclonald use the notion of salience to deal with the selection problem in the task of describing a singje image of a natural oubdoor scene.

TALPSIN exemplifies that plans and goals of an actor may form the underlying structure of narratives and may thus be motivation for text generation. In KDS a representation of what to do in case of fire alamm is transformed into a natural language text. As the initial representation already contains lexical entrios and primitive propositions the thas is to organize this information anew so that it may be expressed in an Jinglish text. Mann and Moore propose rules for combining propositions and reedit the text continuously to produce the final version. 'THX'I penerates para" uraphs as answers to questions about daliabase structure. Mckeown has identified discourse strategies for fulfilling thee conmunicative poals: define, compare, and deseribe. These strategies gaide the generation process in deciding what to say next. MeKeown use: the question to detemine the communicative geal that the text should fulfil. Rescarch of this kind is very important, to clarify the relation between the form of a text and its underlying goals.

One of the domains of IIAM-ANS is the kind of traflic scene which is also used in N $\Lambda O S$, In this domain ILM-ANS deals with primarily with answering, questions about the motions of objects and with overanswering yes/no questions [25]. 'The dialogue component of IIAM-ANS may be connected to NAOS to also allow questions of the user if the generated text was not suflicient for his understanding. An evaluation of the lind of question being asked by a user may help in devising better generation strategies.

KAMP is a system for plinning natural languare utiorances in the domain of task oriented dialogues. 'The planning alsorithm takes the knowledge and beliefs of the hearer into arcount. "This sy. stem shows how a priori beliefs of the hearer nay al:o be integrated in NAOS to generate appropriate referring; phrases.

It would be interesiling to USe a planning component for NAOS which would first delermine all decp cases necessary to maximally restrict the visualized trajectory of an object's motion sequence and then try to distribute the cases to the different verbs used in the description in order to generate smooth text.

\section{y ribliogrephy}

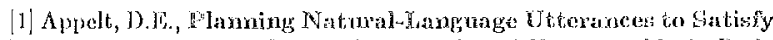
Multiple Conls. SRI Interuational, 'leclunical Note 259, Monlo Park, CA., 1982

[2] Badler, N.Y., 'lemporal Seche Analysis: Conceptual Honcription uf Object Movements. Report $7 \mathrm{R}-80$, Dept. of $\mathrm{CS}$, University of Toronto, 1975

[3] Barwise, J., P'erry, J., Gituationss and Attitudes, Bradford Books, MI'1 Press, 1983
[1] Busemann, S., Surfine Tramtornations during the Generation of Written Corman Sentences. In: B Holc, L. (ocl.), Natural J,angurge Generation Systems. Springer, Berlin, 1984

[5] Condlin, R.J., Mclontald, D.D., Salienes: The Key to the Sebectoon Roblem in Natniral Language Ceneration. COLING-82, 129.135

[6] Davey, A., Discourge Procluetion. A Compnter Model of Some Aapects of a Spealker. Widinburght University Press, 1978

(7) Fillmore, C.I., Scencs-and frames Semantics, [n: Zampolli, A. (ed.), Linguistic Structures Processing. North.flolland, Ansterdam, $1977,55-81$

[8] Goldman, N.M., Coneeptual Genewhion. h!: Schank, R.C. (exl.), Conceptual Information Processing. North. Inolland, 1975, 289-371

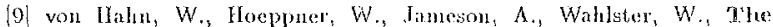
Antatomy of the Natural banguage vialogue Sygtern GAM-

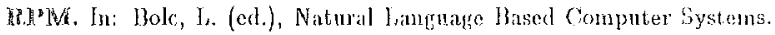
ILanser/McMilian, Müncheni, 1980, 119\%253

[10] Hooppmer, W., Ghristaller, 'll., Marburger, II., Morik, K., Nebel,

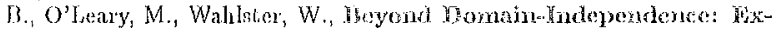
perience with the Development of at Geman Janguaga Acess

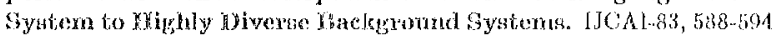
111 Jameson, A., Wahlster, W., Vser Modelling in Anphon Gone:-

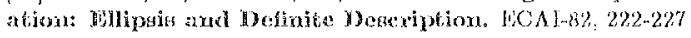

(12) Mann, W.C., Moore, J., Compratere Gemeration of Multiparsgraph Tost. AJCl, $/(1), 1931,17-29$

[13] McDonald, D.D), Watural Gangange Generation as a Con-

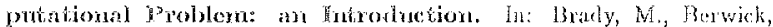
R.C. (eds.), Conpulational Morlelis of Discourse. MT'T l'ress, Cambridge, Mass., 1983, 209-265

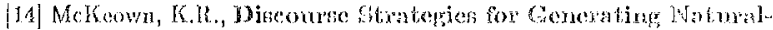
Sanguage 'Text. Artificial Intelligence $27,1985,1-41$

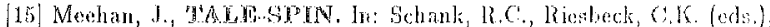
laside Componter Unererstandings: Five Programus plus Miniatures. LWA Ilillsdale, New Jersey, 1981, 197-258

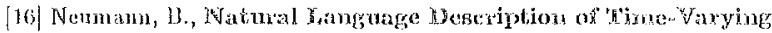
Scenes. In: Waltz, D. (ed.), Advances in Natural Language l'rocessis.s.

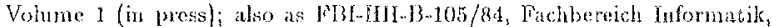
Universitä̈t flamburge, 1984

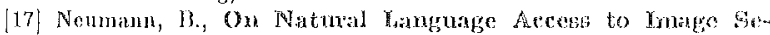
quences: livent Mecognition and Verbalization. Proc. Tirst Confer("Hee on Artificial Intenligence $A_{p}$ plications $(C A \mid \Lambda-84)$, Denver, Colorado, 1984

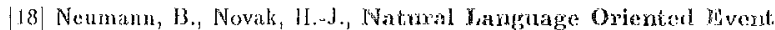

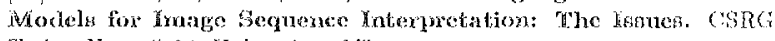
Techn. Note \#34, Whiversity of 'foronto, 1983

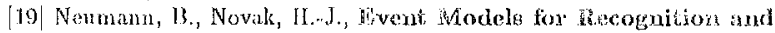

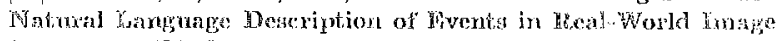
Sequences. IJCAl-83, 724-726

[20] Novak, It.J., A Relational Mateling Sirategy for 'Iemporal

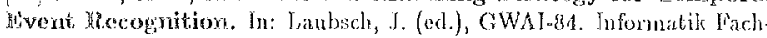
berichte 103, Spritger, 1985, 1095-118

|21. Olson D.R., Isanguage Use for Gommunicatiang, Instrututirg. axid Mhinking. In: liredle, R.O., Carroll, J.13. (eds.), I,anguage Comprehension and the Acquisition of Knowledge. Wakhington, $19 \%$

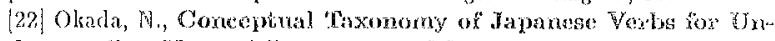

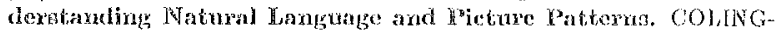
80, $127-135$

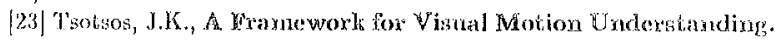
CSRC TIR-114, Uniwersity of 'Toronto, 1980

[2A] 'Tsuji, S., Kuroda, S., Morizono, A., Uorderstamding, a Gixnples Gartoon Film by a Computer Vision Systern. 1JCA1-77, 609-610

[25] Wahlster, W., Marburqer, H., Janeson, A., Busemann, $\mathfrak{S}^{3}$, Overan-

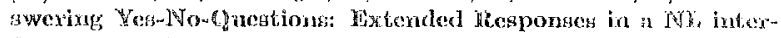
face to a Vinton Syttem. IJCAI-83, 643-646 